\title{
Monte-Carlo simulation of gamma-cameras using GEANT
}

\author{
J. Berthot ${ }^{2}$, V. Breton ${ }^{2}$, P. Brette ${ }^{3}$, N. Giokaris ${ }^{4}$, D. Lazaro ${ }^{2}$, J. Maublant ${ }^{5}$,L. Méritet ${ }^{2}$ \\ ${ }^{2}$ LPC, Campus des Cézeaux, 63177 Aubière cedex, France \\ ${ }^{3}$ Clérad, 16 Avenue de l'agriculture, 63000 Clermont-Ferrand, France \\ ${ }^{4}$ Institute of Accelerating Systems and Applications, Athens, Hellas \\ ${ }^{5}$ Service de Médecine Nucléaire, Centre Jean Perrin, 63000 Clermont-Ferrand, France
}

\begin{abstract}
There is a growing interest among nuclear and particle physicists for the application of the detection techniques used in subatomic physics to medical imaging. As well, the code used to simulate the response of these detectors to ionizing particles, named GEANT, can be applied to simulate medical imaging devices. GEANT allows a complete Monte-Carlo treatment of the attenuation by body parts and of the generation and tracking of optical photons from scintillation inside the crystal. We used GEANT to simulate a high resolution gamma camera for small animal imaging. Our study shows a significant correlation between the depth of a source in the body and the energy spectrum of the photons detected in the gamma-camera.
\end{abstract}

\section{INTRODUCTION}

A reliable Monte-Carlo simulation is of utmost importance to evaluate the performances of the gamma cameras and search for compromises between resolution and sensitivity. Usually, the research teams working on their own gamma-camera prototype have been developing their own simulation code which implies an increased risk of bugs and a reduced transportability.

For more than 2 decades, the subatomic physics community around the world has been using the MonteCarlo code GEANT to describe the interaction of ionizing particles with matter. The GEANT code was developed at CERN to deal with the increasing scale and complexity of the high energy physics experiments. Simulation studies became essential to

design and optimize the detectors

develop and test the reconstruction and analysis programs and interpret the experimental data.

The interest of using GEANT to simulate gammacameras is threefold :

All the physics processes involved from the emission of the radioisotope to the creation of the image in the gamma-camera are described in GEANT or can be added.

The code is highly reliable because it is used extensively around the world.

The code is a standard in high energy physics and therefore there is abundant documentation and many experts.
The first version of GEANT was written in 1974 as a bare framework which initially emphasized tracking of a few particles per event through relatively simple detectors. The system has been developed continuously over the years thanks to the devoted and constant collaboration of physicists around the world. There are today two versions :

GEANT version 3 [1] was written in Fortran by a team at CERN as a complete update from previous versions. Concerning medical imaging applications, electromagnetic processes are simulated down to $10 \mathrm{keV}$.

GEANT version 4 is a completely new code developed within an international collaboration of about 100 scientists participating in more than 10 experiments in Europe, Russia, Japan, Canada and the United States. To extend its use toward medical physics and space physics, very low energy processes down to tens of electron-volts are included. Written in object-oriented language, the GEANT4 source code is freely available [2].

This paper presents the results we have obtained using GEANT321, the last version of GEANT in fortran, for imaging low energy gamma rays from ${ }^{99 \mathrm{~m}} \mathrm{Tc}$. We studied the reliability of the simulation to reproduce the performance (spatial resolution, sensitivity) of a camera which is presently under development for small animal imaging [5]. Once the simulation was validated, we studied the correlation between the depth of the source inside the body and the shape of the energy spectrum of photons detected in the camera.

\section{Structure of The Simulation Code}

We are going to describe the different parts of the simulation code. Figure 1 shows the different elements of the geometry: source(s), body, collimator, crystal and photomultipliers.

\section{A. Sources}

The sources are located inside the body. Their number, size, shape and position are parameters adjustable at execution time. We considered only ${ }^{99 \mathrm{~m}} \mathrm{Tc}$ isotropic sources emitting $140 \mathrm{keV}$ photons. 


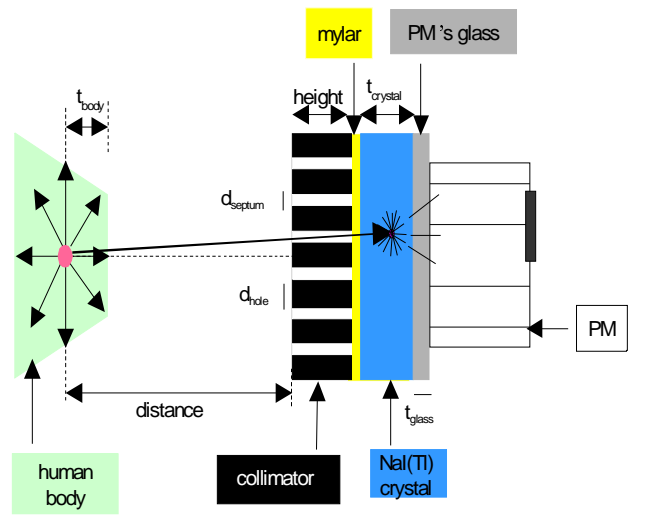

Figure 1: The different elements of the geometry.

\section{B. Body}

The camera we simulate is designed for small animal imaging. However, our focus is mammography. Therefore, body was simulated by a trapezoid of variable thickness. Breast tissue atomic composition was taken from NIST web site [2]. In the body, photons emitted from the sources undergo Compton scattering and photoelectric effect. Rayleigh scattering was neglected.

\section{Collimator}

Among the gamma cameras on the market, many options for the geometry of the collimators are considered depending upon the specific needs. For the purpose of validating the simulation, we considered 2 widely used configurations : parallel hole and pin-hole geometry. For parallel hole collimators, we considered hexagonal cells separated by thin foils. Among the heavy materials used for collimators, we chose lead.

The size of the holes and the septa, the thickness of the collimator as well as its material are free parameters of the simulation.

\section{Crystal}

One of the hardest issues for the Monte-Carlo simulation of gamma cameras concerns the treatment of optical photons generated by scintillation in the doped $\mathrm{NaI}(\mathrm{Tl})$ crystal. Indeed, about 40000 optical photons per $\mathrm{MeV}$ of energy are generated. Each photon must be followed in its travel inside the crystal to the photomultipliers entrance window. GEANT321 allows a reliable description of optical photons transport, reflection and refraction. It has however no treatment of scintillation. We therefore added our own generation of optical photons : when GEANT stops tracking photons and electrons inside the crystal because they fell below the energy threshold $(\approx 10 \mathrm{keV}), 3 \mathrm{eV}$ optical photons are generated isotropically at stopping point : 40000 optical photons per $\mathrm{MeV}$ incident energy are then tracked by GEANT.

\section{E. Light collection}

Light collection is probably the weakest point of our simulation because we did not enter into the details of describing the electronic shower in the photomultipliers. In this work, the number of photons collected by the PM's was adjusted varying the quantum efficiency. Photomultipliers output was calibrated on the photoelectric peak.

\section{V ALIDATION OF THE SIMULATION}

We first checked the validity of the simulation by comparing the collimator performances to the analytical formulas. The energy resolution of the camera was tuned by varying the crystal thickness and the quantum efficiency of the photomultipliers.

\section{A. Collimators intrinsic resolution and sensitivity}

We studied two types of collimators. This section presents our results compared to the analytical formulas concerning the intrinsic resolution and the sensitivity of the simulated collimators. The intrinsic resolution of a collimator is defined as the FWHM of the spatial distribution of photons passing through the collimator. Three parameters characterize the geometry of a parallel hole collimator : the holes diameter, the septa diameter and the collimator height (see figure 1). For 500000 photons generated at the source located $10 \mathrm{~cm}$ in front of the collimator, the simulation results are in excellent agreement with analytical formulas as shown in table 1 . The same results are obtained for the pin-hole collimator.

Table 1

Collimator resolution : analytical formula vs. simulation

\begin{tabular}{|l|c|c|}
\hline Collimator & Parallel hole & Pin hole \\
\hline $\begin{array}{l}\text { Hole diameter } \\
(\mathrm{mm})\end{array}$ & 1,9 & 4,0 \\
\hline $\begin{array}{l}\text { Septum diameter } \\
(\mathrm{mm})\end{array}$ & 0,5 & 3,0 \\
\hline Height $(\mathrm{cm})$ & 3,0 & 5,0 \\
\hline $\begin{array}{l}\text { Simulated } \\
\text { resolution } \\
(\mathrm{mm})\end{array}$ & 6,5 & 5,2 \\
\hline $\begin{array}{l}\text { Analytical } \\
\text { resolution } \\
(\mathrm{mm})\end{array}$ & 6,5 & \\
\hline
\end{tabular}

The sensitivity is the ratio of the number of incident photons emitted by the ${ }^{99 \mathrm{~m}} \mathrm{Tc}$ source and transmitted by the collimator, to the number of photons emitted by the ${ }^{99 \mathrm{~m}} \mathrm{Tc}$ source. The simulation conditions are the same as previously. We compare again simulated and analytical sensitivities for 500000 photons generated. 
Table 2

Collimator sensitivity : analytical formula vs. simulation

\begin{tabular}{|l|c|c|}
\hline Collimator & Parallel hole & Pin hole \\
\hline $\begin{array}{l}\text { Simulated } \\
\text { sensitivity (\%) }\end{array}$ & 0,011 & 0,013 \\
\hline $\begin{array}{l}\text { Analytical } \\
\text { sensitivity (\%) }\end{array}$ & 0,013 & 0,010 \\
\hline
\end{tabular}

Table 2 shows the good agreement between simulated and analytical values for the parallel hole and the pin-hole geometry.

\section{B. Energy resolution}

Most of the photons entering the crystal are stopped in the crystal through a photoelectric effect. For those photons that did not scatter inside the body, energy deposit in the crystal is therefore $140 \mathrm{keV}$. This energy is converted in optical photons. The output signal from the photomultipliers is proportional to the number of optical photons collected.

For a given collimator, energy resolution characterizes the chain crystal - photomultipliers. To take out the impact of the collimator, we considered a gamma camera with a pin-hole collimator and a ${ }^{99 \mathrm{~m}} \mathrm{Tc}$ source at $10 \mathrm{~cm}$ in front of it emitting photons along the camera axis. Figure 2 shows the energy spectrum of the photons entering the crystal (in black) and reconstructed from PM's output (in white) for 2 different quantum efficiencies : $10 \%$ and $20 \%$.
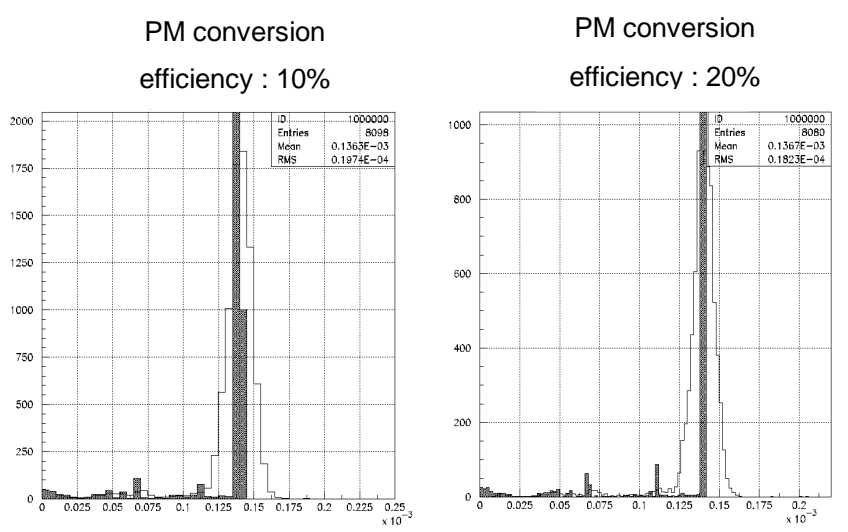

Figure 2 : energy spectra of the photons entering the crystal (in black) and reconstructed from PM's output (in white) for 2 different quantum efficiencies : $10 \%$ and $20 \%$.

The energy resolution is the FWHM of the distribution of the number of optical photons for the events in the photoelectric peak. We find an energy resolution of 13,6\% for a PM's quantum efficiency of $10 \%$, and an energy resolution of $10,9 \%$ for a PM's quantum efficiency of $20 \%$.

We studied the energy resolution for various thicknesses of crystal and a $10 \%$ quantum efficiency. The results are presented in Table 3.
Table 3

Energy resolution vs. crystal thickness

\begin{tabular}{|c|c|}
\hline Crystal thickness $(\mathrm{mm})$ & Energy resolution $(\%)$ \\
\hline 2 & 10,4 \\
\hline 4 & 12,0 \\
\hline 6 & 13,6 \\
\hline 8 & 14,6 \\
\hline 10 & 15,9 \\
\hline
\end{tabular}

The energy resolution obtained by simulation is compatible with the experimental values usually observed and increases with the crystal thickness.

All these results contribute to say that GEANT321 is a valid simulation tool to evaluate gamma cameras performances. We chose to study with GEANT the information the Compton peak could bring on the depth of the source inside the body.

\section{Attenuation Ratio}

The standard procedure to reconstruct the image of a body part from the output of a gamma camera is to select the events in the photoelectric peak. These photons are characterized by the fact they did not undergo Compton scattering in the body. We call $\mathrm{P}_{\mathrm{i}}$ the probability for a photon to enter the crystal after scattering $\mathrm{i}$ times in the body. It is easy to see that the probability for a photon to have scattered at least once before entering the crystal is :

$$
\sum_{i=1}^{n} P_{i}
$$

By definition of $\mathrm{P}_{\mathrm{i}}$, the probability not to have interacted before entering the crystal is:

$$
1-\sum_{\mathrm{i}=\mathbf{1}}^{\mathrm{n}} \mathrm{P}_{\mathrm{i}}
$$

We define the ratio $\mathrm{R}$ of the number of events in the photoelectric peak to the number of events in the Compton peak. If the energy resolution of the detector is good enough to separate the two peaks, arguments developed above suggest that $\mathrm{R}$ is proportional to

$$
\frac{1-\sum_{i=1}^{n} P_{i}}{\sum_{i=1}^{n} P_{i}}
$$

$\mathrm{P}_{\mathrm{i}}$, probability for a photon to have undergone Compton scattering i times before leaving the body and entering the crystal, depends on the Compton scattering cross section and the body thickness $t_{\text {body. }}$ It can be written as

$$
\mathrm{P}_{\mathrm{i}}=\alpha_{\mathrm{i}} \mathrm{t}_{\text {body }}^{\mathrm{i}}
$$


where the coefficient $\alpha_{i}$ contains the information pertaining to the Compton cross section integrated on the scattering angle.

The ratio $\mathrm{R}$ is therefore proportional to

$$
\frac{1-\sum \alpha_{\mathrm{I}} \mathrm{t}_{\text {body }}^{\mathrm{i}}}{\sum \alpha_{\mathrm{i}} \mathrm{i}_{\text {body }}^{\mathrm{i}}}
$$

In the limit $t_{\text {body }} \rightarrow 0, \mathrm{R}$ should behave as $1 / \mathrm{t}_{\text {body }}$ and therefore depend strongly on $t_{\text {body }}$.

We have simulated the variation of the ratio $\mathrm{R}$ with the depth of the source inside the body. We have defined windows of energies for the 2 energy spectra available : true energy of photons entering the crystal and reconstructed energy from PM's output. Results are shown on figure 3 . The $1 / t_{\text {body }}$ dependence is well reproduced by the simulation.

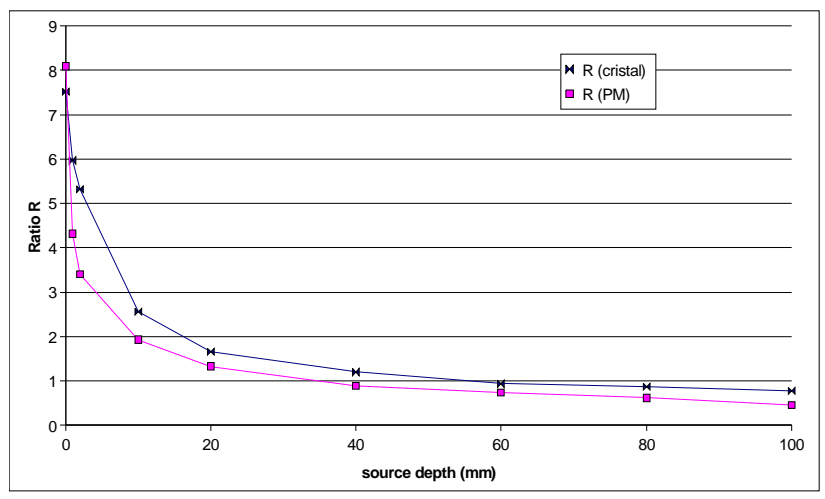

Figure 3: Evolution of the ratio $\mathrm{R}$ in the $\mathrm{NaI}(\mathrm{Tl})$ crystal and in the PM vs. source depth.

This result has to be compared to an experimental observation of the same effect [6].

\section{Conclusion}

According to the very good agreement between simulation results and theoretical values, we can say GEANT321 constitutes a promising simulation tool for gamma-cameras. The results obtained concerning collimator resolution and sensitivity allow to validate the simulation, although certain parts of it still deserve to be developed. So we have at our disposal a complete and reliable simulation code.

With GEANT321, we focussed on the possibility to use the Compton peak information to determine the source depth. Our simulation stresses the fact that the ratio $\mathrm{R}$ of the number of events in the photoelectric peak to the number of events in the Compton peak goes with the inverse of the source depth in the body. Provided events in the Compton peak could be collected together with the events in the photoelectric peak, the information on the source depth could be obtained without additional exposure of the patient.

\section{REFERENCES}

[1] GEANT3, Detector description and simulation tool, Application Software Group, CERN Geneva, Switzerland, 1993

[2] GEANT4 web site : wwwinfo.cern.ch/asd/geant4/geant4.html

[3] NIST web site: www.physics.nist.gov/PhysRefData

[4] Development of a High Resolution Spect Gamma Camera for Small Animals Imaging, S.C. Archimandritis et al, IEEE 2000

[5] Small Gamma-Ray Imager Based on a Position Sensitive Photomultiplier Tube, A. Lebedev, S. Majewski, D. Steinbach and A. Weisenberg, submitted to Nuclear Instruments and Methods in Physics Research.

[6] M.A. Gambaro et al, contribution to European Association of Nuclear Medicine Congress, Paris 2000 